\title{
Structural neuroimaging correlates of social deficits are similar in autism spectrum disorder and attention-deficit/hyperactivity disorder: analysis from the POND Network
}

\author{
Danielle A. Baribeau', Annie Dupuis², Tara A. Paton ${ }^{3}$, Christopher Hammill ${ }^{4}$, Stephen W. Scherer ${ }^{3,5}$, Russell J. Schachar ${ }^{1,6}$, \\ Paul D. Arnold ${ }^{7}$, Peter Szatmari 1,6,8, Rob Nicolson ${ }^{9}$, Stelios Georgiades ${ }^{10}$, Jennifer Crosbie ${ }^{1,6}$, Jessica Brian ${ }^{11,12}$, \\ Alana laboni $^{12}$, Azadeh Kushki ${ }^{12,13}$, Jason P. Lerch ${ }^{4,14}$ and Evdokia Anagnostou ${ }^{11,12}$
}

\begin{abstract}
Autism spectrum disorder (ASD), attention-deficit/hyperactivity disorder (ADHD), and obsessive-compulsive disorder (OCD) have been associated with difficulties recognizing and responding to social cues. Neuroimaging studies have begun to map the social brain; however, the specific neural substrates contributing to social deficits in neurodevelopmental disorders remain unclear. Three hundred and twelve children underwent structural magnetic resonance imaging of the brain (controls $=32, \mathrm{OCD}=44, \mathrm{ADHD}=77, \mathrm{ASD}=159$; mean age $=11$ ). Their social deficits were quantified on the Social Communication Questionnaire (SCQ) and the Reading the Mind in the Eyes Test (RMET). Multivariable regression models were used to examine the structural neuroimaging correlates of social deficits, with both a region of interest and a whole-brain vertex-wise approach. For the region of interest analysis, social brain regions were grouped into three networks: (1) lateral mentalization (e.g., temporal-parietal junction), (2) frontal cognitive (e.g., orbitofrontal cortex), and (3) subcortical affective (e.g., limbic system) regions. Overall, social communication deficits on the SCQ were associated with thinner cortices in the left lateral regions and the right insula, and decreased volume in the ventral striatum, across diagnostic groups $(p=0.006$ to $<0.0001)$. Smaller subcortical volumes were associated with more severe social deficits on the SCQ in ASD and ADHD, and less severe deficits in OCD. On the RMET, larger amygdala/hippocampal volumes were associated with fewer deficits across groups. Overall, patterns of associations were similar in ASD and ADHD, supporting a common underlying biology and the blurring of the diagnostic boundaries between these disorders.
\end{abstract}

\section{Introduction}

Social deficits are a defining feature of autism spectrum disorder (ASD), but also frequently affect children with attention-deficit/ hyperactivity disorder $(\mathrm{ADHD})^{1}$, and can occur in obsessive-compulsive disorder (OCD) as

\footnotetext{
Correspondence: Danielle A. Baribeau (danielle.baribeau@mail.utoronto.ca) 'Department of Psychiatry, University of Toronto, Toronto, ON, Canada ${ }^{2}$ Clinical Research Services, The Hospital for Sick Children, and the Dalla Lana School of Public Health, University of Toronto, Toronto, ON, Canada Full list of author information is available at the end of the article.
}

well $^{2-5}$. Increasingly, recognition of the overlapping and related nature of both the symptoms and the biology of different neurodevelopmental disorders ${ }^{6-8}$ has led to a call for research that spans diagnostic boundaries, and focuses instead on dimensions of psychopathology ${ }^{9}$.

Decades of neuroimaging research have begun to delineate the neural substrates of sociality. Different theoretical models of the social brain have been put forward from meta-analyses ${ }^{10,11}$ and reviews of the literature ${ }^{12-14}$ (summarized in Supplementary Table 1). Across models, 
three functional/structural groups emerge. Brain regions hypothesized to be involved in mentalization and empathy (group 1), cluster along the midline and lateral aspects of the brain, including the temporal-parietal junction (TPJ), superior temporal gyrus/sulcus (STS/STG), dorsal medial prefrontal cortex, temporal poles, and the posterior cingulate. Anterior and prefrontal regions (group 2), including the anterior and dorsal cingulate, orbital frontal cortex, and the dorsal and ventral lateral prefrontal cortices, may contribute to executive function and cognitive control over affective and social processes. Deeper cortical and subcortical structures (group 3), including the insula, amygdala, hippocampus, and the dorsal and ventral striatum, are more central to affective responding, memory, and social reward processing (Supplementary Table 1).

Structural neuroanatomical differences in many of these regions have been detected in individuals with OCD, ASD, and ADHD compared to controls ${ }^{8,15-21}$. For example, recent meta-analyses suggest increased frontal lobe thickness in $\mathrm{ASD}^{20}$, thinner temporal/parietal thickness in ASD and $\mathrm{OCD}^{20,22}$, and smaller subcortical volumes in $\mathrm{ASD}$ and $\mathrm{ADHD}^{20,23}$. The extent to which social deficits may differentially localize to specific brain regions/networks in different disorders is unclear, however ${ }^{24}$. In ASD, social deficits have been associated with both larger ${ }^{25-29}$ and smaller ${ }^{27,30,31}$ cortical measurements, particularly in frontal and temporal-parietal regions. In ADHD, social deficits were associated with greater overall cortical gray matter volume ${ }^{32}$ and smaller left caudate volumes ${ }^{33}$.

Cortical gray matter volume is a product of cortical thickness and surface area. Recent work has provided evidence that cortical thickness and cortical surface area measurements are under distinct genetic influences ${ }^{34}$, and follow unique developmental timelines ${ }^{35,36}$, necessitating that they be studied independently. Cortical thickness measurements on magnetic resonance imaging (MRI) are thought to reflect the underlying cortical microstructure, involving the number and organization of cortical neurons, neuronal dendritic arborization, the number and size of glial cells, and to some extent the maturation of the adjacent white matter ${ }^{37}$. Cortical thickness, including the timing and rate of cortical thinning, has been a major area of study across neurodevelopmental disorders, particularly in $\mathrm{ASD}^{24}$.

The association between brain (i.e., cortical thickness/ subcortical volume) and behavior (i.e., social deficits) was the focus of the following study, in efforts to identify and compare the neural substrates of sociality across disorders. Given that the specific behavioral dimensions that contribute social impairments may vary across neurodevelopmental disorders (e.g., impaired mentalization in $\mathrm{ASD}^{38}$, executive function in $\mathrm{ADHD}^{39}$, and reward processing in $\left.\mathrm{OCD}^{40}\right)$, one hypothesis is that the neutral substrates of sociality will also differ by diagnosis (e.g., primarily lateral mentalization regions in ASD, frontal cognitive regions in ADHD, and subcortical regions OCD). An alternative hypothesis is that the brain regions associated with social deficits will span diagnostic boundaries. In children with ASD, ADHD, and OCD, for example, white matter fractional anisotropy correlated with adaptive functioning abilities, irrespective of diagnosis ${ }^{8}$. Neuroimaging analyses comparing the structural neuroanatomical correlates of social deficits across children with ASD, ADHD, or OCD have not yet been performed.

To address this knowledge gap, we first compared cortical thickness/subcortical volume measurements in social brain regions across a group of children with ASD, ADHD, OCD, or controls. Next, we examined how cortical thickness/subcortical volume corresponded with social deficits across disorders. We hypothesized that social deficits would correlate with structural anatomy, irrespective of diagnosis.

\section{Methods \\ Participants}

Participants were recruited via the Province of Ontario Neurodevelopmental Disorders Network, across four Centers in Ontario, Canada (Holland Bloorview Kids Rehabilitation Hospital, Toronto; The Hospital for Sick Children, Toronto; McMaster Children's Hospital, Hamilton; and Lawson Health Research Institute, London). Controls were recruited through advertising in public transit, in hospitals, and on social media. Inclusion criteria were age $<18$ years, and a clinical diagnosis of ADHD, ASD, or OCD. Controls had no developmental diagnosis, and no first-degree family history of such. Participants were recruited into the study based on their primary psychiatric diagnosis; potential comorbid symptoms/traits were captured on symptom surveys (described below). Standardized research assessments confirmed the primary clinical diagnosis using established metrics (the Autism Diagnostic Observation Schedule $-2^{41}$, the Autism Diagnostic Interview-Revised (ADI- $\mathrm{R})^{42}$, the KiddieSchedule for Affective Disorders and Schizophrenia ${ }^{43}$, the Parent Interview for Child Symptoms ${ }^{44}$, and the Children's Yale-Brown Obsessive-Compulsive Scale ${ }^{45}$ ). Research ethics board approval was obtained at each institution.

\section{Social metrics: The Social Communication Questionnaire and the Reading the Mind in the Eyes Test}

The Social Communication Questionnaire (SCQ) is an established 39-item measure that quantifies risk for ASD through parent/caregiver report on social abilities and behaviors ${ }^{46}$. For our analyses, we focused on the 28 items assessing current and past social communication/ 
interaction skills, excluding items on repetitive behaviors. The Reading the Mind in the Eyes Test (RMET) is a validated social perception task, in which study participants are asked to label the emotion/mental state in a still image of human eyes ${ }^{47}$. This metric quantifies social perception abilities. Participants in this study completed the child version of the RMET, which included at total of 28 items $^{48}$. For simplicity and congruence with the SCQ, we examined the number of incorrect RMET items. Therefore, for both measures, higher scores indicate greater social deficits.

\section{Other measures}

To characterize the study sample, ADHD symptoms were quantified across groups using the Child Behavior Checklist (CBCL) ADHD subscale. This is a parent report measure that yields age- and sex-normed $T$-scores. $T$ scores $>65$ are suggestive of elevated symptomatology ${ }^{49}$. To quantify OCD symptoms, the Toronto ObsessiveCompulsive scale was used. This is a parent report measure, where items are scored -3 to +3 ; total scores $>0$ discriminated OCD cases from controls in a community sample $^{50}$.

\section{MRI and image analysis}

All structural MRI data were collected at the Hospital for Sick Children in Toronto, between June 2012 and July 2017. The majority of scans (74\%) took place on the 3-Tesla Siemens Trio TIM; a hardware upgrade to the Siemens Prisma scanner took place in June of 2015 (this affected $26 \%$ of the ADHD sample, $24 \%$ of the ASD sample, $62 \%$ of controls, and $7 \%$ of the OCD sample).

Cortical thickness measures were extracted from T1-weighted images using the CIVET pipeline (version 2.1.0 $)^{51}$ The CIVET pipeline applies a non-uniformity correction on the images ${ }^{52}$ followed by stereotaxic registration to the Montreal Neurologic Institute (MNI ICBM152) template (non-linear sixth-generation target) ${ }^{53,54}$. Next, brains were masked, extracted, and classified into gray matter, white matter, and cerebrospinal fluid, which were used to generate gray and white matter surfaces $^{55-59}$. A surface-diffusion kernel was applied ${ }^{60}$, and regions were registered to the automated anatomical labeling atlas ${ }^{61-63}$. Cortical thickness measurements were taken from the distance between the two smoothed surfaces $^{64,65}$. Quality assurance was assessed at the time of the scan for motion artifact, and was analyzed through the CIVET quality control (QC) analysis pipeline. Scans that were flagged on the $\mathrm{QC}$ analysis were manually reviewed for quality, and potentially excluded. Thickness measurements from the automated anatomical labeling atlas regions were grouped into regions of interest to reflect the three primary social neural networks ${ }^{10,12-14}$ (see Supplementary Table 1). We computed the vertex-wise thickness for each combined region of interest, and also examined the individual vertices across the whole brain. Subcortical structures were segmented using multiple automatically generated templates (MAGeT) and their volumes determined from the segmentation results ${ }^{66}$. For the region of interest analysis, we examined the association between mean thickness/volume measurements and social deficits in both the left and right hemispheres combined, except where previous data suggested significant lateralization effects (i.e., the temporal-parietal region ${ }^{24,29,67}$, the amygdala $^{68,69}$, the dorsal striatum ${ }^{33}$, and the insula $\left.{ }^{70}\right]$.

\section{Statistical analyses}

Statistical analyses were performed using SAS (University Edition) version 9.4, and $\mathrm{R}$ version 3.5.1.

As a preliminary analysis, we initially tested for diagnostic differences in brain structure for the combined social regions of interest, as well as mean whole-brain cortical thickness and volume. For this, we used a linear regression model to estimate the least-squares (LS) mean cortical thickness (in $\mathrm{mm}$ ) by region, or mean volume $\left(\mathrm{mm}^{3}\right)$ for subcortical structures, treating diagnosis (ASD, ADHD, OCD, or control) as a categorical predictor variable, while also including age, sex, and hardware upgrade as covariates to account for their effects.

Next, we used a logistic regression model to examine the proportion of items selected on the social measures (SCQ score/28 items, or RMET incorrect score/28 items) as the dependent variable, predicted by each millimeter increase in cortical thickness, or $0.1 \mathrm{~cm}^{3}$ increase in subcortical volume. Regions were examined in the three structural/functional groups. Odds ratios (ORs) are presented; ORs $>1$ indicate greater deficits with increasing size, whereas ORs $<1$ indicate fewer social deficits with increasing size. We included age, sex, hardware upgrade, and diagnosis as covariates, while also testing for interactions between thickness/volume and diagnosis. Where the thickness/volume-by-diagnosis interaction term was non-significant $(p>0.05)$, the interaction term was dropped from the model and ORs were presented across all groups combined. Where the interaction term was significant, ORs were presented for the diagnostic groups separately. Logistic regression analysis was chosen given that scores on the SCQ and RMET have a ceiling/floor, and follow a binomial distribution. Results are presented without correction for multiple comparisons in order to convey overall patterns; associations that remained significant after a false discovery rate (FDR) correction ${ }^{71}$ are indicated in the text/tables.

As a secondary analysis, the association between cortical thickness and social behavior was examined using a whole-brain vertex-wise approach, with the same logistic regression models applied to 70,000 vertices across the entire brain. ORs for vertices that remained significant 
after an FDR correction $(q<0.05)$ are presented graphically. The proportion of significant voxels, and the number of significant peaks (i.e., significant statistical maxima within a five vertex radius) per region of interest are also presented.

\section{Results}

\section{Demographic characteristics}

Of 343 initial scans, 31 (9\%) were excluded due to low quality. Demographic data for all remaining participants are presented in Table 1. As expected, the ASD group had the highest level of social deficits on the SCQ and the RMET. All groups separated in terms of ADHD symptoms on the CBCL (ADHD > ASD > OCD > controls). The ADHD and control groups were similar in terms of OCD symptom severities. Correlations between behavioral measures are presented in Supplementary Table 2.

\section{No diagnostic differences in social brain structures between groups}

The multivariable predicted LS means for the regions of interest for each diagnostic group are presented in Supplementary Table 3 . There were no significant differences in brain structure between diagnostic groups with the exception of the hippocampus, where diagnosis had a nominal effect on hippocampal volumes $(F=4.10, p=$ 0.007 , non-significant after FDR correction). On pairwise comparisons, the OCD group had larger mean hippocampal volumes. Also adjusting for whole-brain volume yielded a less significant main effect of diagnosis on hippocampal volume $(F=2.62, p=0.05)$; other structures remained non-significant.

\section{Structural neuroimaging correlates of social communication deficits are similar in ASD and ADHD; OCD has some unique features}

Contrary to our initial hypotheses, the association between social brain region size and social deficits as measured by the SCQ varied significantly by diagnosis in the right lateral regions, frontal regions, left and right amygdala, hippocampus, bilateral dorsal striatum, and the left insula $(p<0.008$ for all region-by-diagnosis interaction terms) (Table 2). Distinct patterns emerged for each diagnostic group (Fig. 1). In the control group, thinner lateral regions and insula, and smaller left amygdala and ventral striatal volumes were associated with higher SCQ scores, therefore greater social deficits $(\mathrm{OR}<1.0)$. Conversely, in the OCD group, while thinner cortical regions were associated with greater social deficits, smaller subcortical volumes and left insular thickness were associated with fewer social deficits (OR >1.0). In ADHD and ASD, thinner cortical and smaller subcortical structures were associated with increased social deficits in a similar pattern. Findings remained significant after FDR correction
(Table 2). The distinct subcortical pattern (e.g., OR $>1.0$ in OCD, and $<1.0$ in ASD and ADHD) persisted after also adjusting for comorbid OCD symptoms (on the TOCS) or ADHD symptoms (on the CBCL-ADHD subscale) (data not shown). Across all findings, wide confidence intervals and significant spread on scatter plots (shown in Supplementary Figure 1) suggested a high degree of heterogeneity in patterns of association.

\section{Social perception deficits inversely correlated with amygdala and hippocampal volumes across diagnoses}

On the RMET, region-by-diagnosis interaction terms were only significant in the right and left lateral regions $(p=0.02$ and 0.04$)$ and the right insula $(p=0.02)$, although findings became non-significant after FDR correction. For these regions, interaction terms were kept in the model to convey patterns, however. Specifically, the ASD group showed a significant association (thinner cortex $=$ greater deficits) and other groups were nonsignificant (Table 3, Fig. 2 (top panel)). Elsewhere, larger left amygdala volume $(p<0.0001)$, right amygdala volume $(p=0.003)$, and hippocampal volumes $(p=0.001)$ were associated with fewer social deficits across groups. As a sensitivity analysis, we also adjusted for IQ in the model, given data showing that RMET scores vary with cognition ${ }^{1}$. Adjusting for IQ resulted in a similar pattern of associations (Supplementary Table 4).

\section{Whole-brain vertex-wise analysis}

On whole-brain vertex-wise analysis, for the SCQ, patterns of associations were similar as found with the region of interest approach (Fig. 3, Supplementary Tables 5 and 6). Specifically, in the right lateral regions, $12 \%$ of voxels were significant in ADHD, 24\% in ASD, and 87\% in OCD, where generally thicker cortices were associated with fewer social deficits. For the left lateral regions, group differences were less pronounced (17\% in ADHD, $22 \%$ in ASD, and 30\% in OCD). Similarly, for the combined frontal regions, $18 \%$ of voxels were significant in ADHD, $39 \%$ in ASD, and 70\% in OCD. The number of statistical peaks within a five vertex radius generally mirrored the proportion of significant voxels (Supplementary Tables 5 and 6). No associations remained significant after FDR correction for the control group. For OCD, vertex-wise associations were more lateralized than with the region of interest approach. The majority of the right hemisphere showed significant associations (where thicker cortex $=$ fewer deficits); there were reciprocal effects (thicker cortex $=$ greater social deficits) in the left insula (see Fig. 3). For the RMET, participants with ASD had fewer social deficits with thicker cortices in both the left (16\% of voxels) and right (24\% of voxels) lateral regions, with scattered frontal associations (30\% of voxels), partially consistent with patterns in the region of interest analyses 


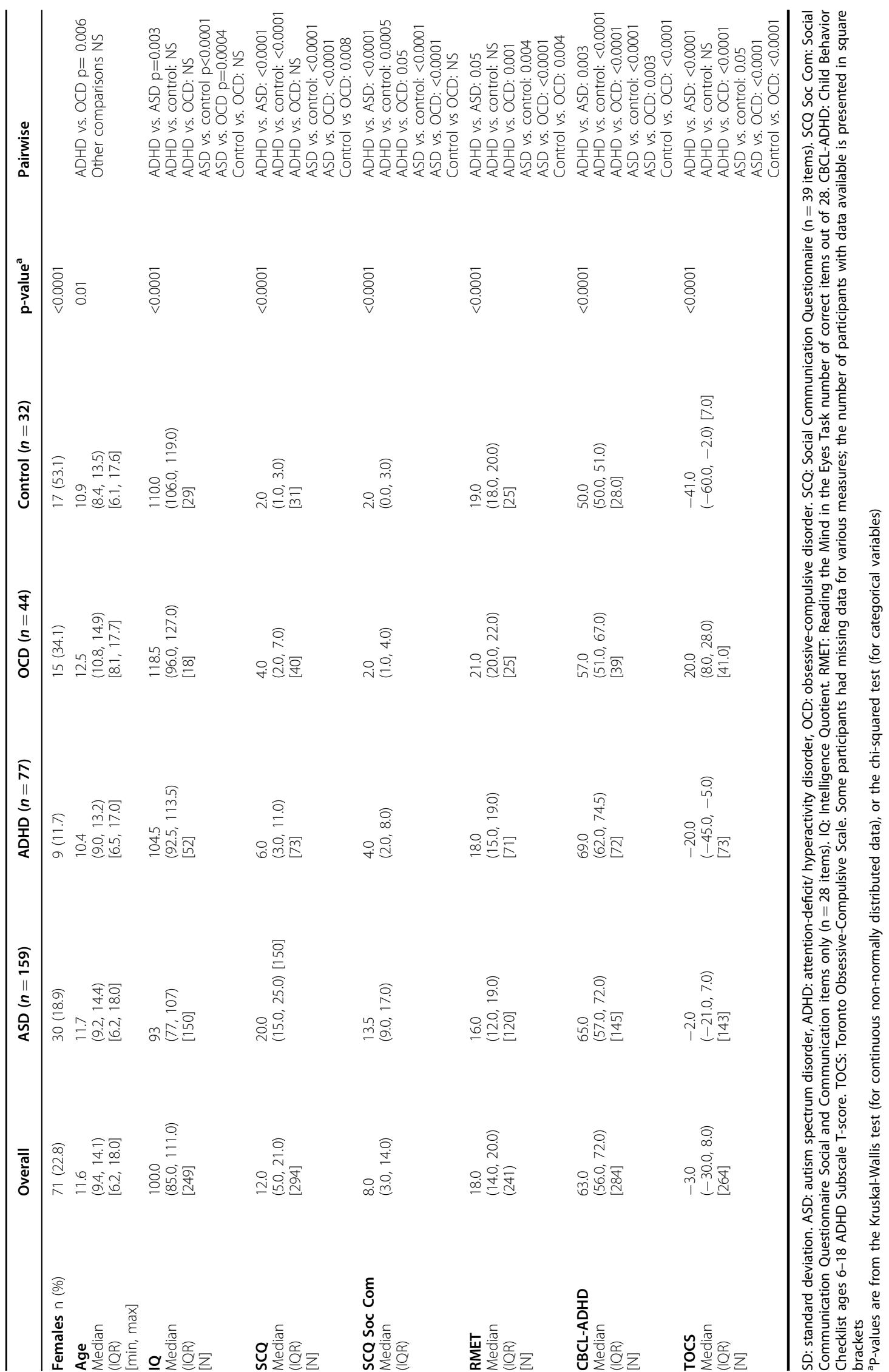


Table 2 Association between social regions of interest and social deficits on the SCQ

\begin{tabular}{|c|c|c|c|c|c|c|}
\hline \multirow[t]{2}{*}{ Region } & \multicolumn{2}{|l|}{ Wald $X^{2}, p$ value } & \multirow[t]{2}{*}{ OR ASD, $95 \% \mathrm{Cl}$} & \multirow[t]{2}{*}{ OR ADHD, $95 \% \mathrm{Cl}$} & \multirow[t]{2}{*}{ OR OCD, $95 \% \mathrm{Cl}$} & \multirow[t]{2}{*}{ OR Control, $95 \% \mathrm{Cl}$} \\
\hline & Region & Region $\times \mathrm{Dx}$ & & & & \\
\hline \multicolumn{7}{|c|}{ Lateral mentalization regions } \\
\hline Left lateral regions & $12.21,0.0005$ & & $0.55,0.39-0.77$ & $0.55,0.39-0.77$ & $0.55,0.39-0.77$ & $0.55,0.39-0.77$ \\
\hline Right lateral regions & & $16.62,0.008$ & $0.71,0.48-1.03$ & $0.54,0.27-1.07$ & $0.17,0.083-0.33$ & $0.12,0.03-0.57$ \\
\hline \multicolumn{7}{|l|}{ Frontal cognitive regions } \\
\hline Frontal regions & & $18.85,0.0003$ & $0.49,0.30-0.79$ & $0.44,0.18-1.05$ & $0.03,0.01-0.10$ & $0.29,0.55-1.48$ \\
\hline \multicolumn{7}{|c|}{ Deeper and subcortical affective regions } \\
\hline Left amygdala' & & $27.53,<0.0001$ & $0.92,0.87-0.98$ & $0.79,0.73-0.87$ & $1.20,1.03-1.41$ & $0.60,0.41-0.87$ \\
\hline Right amygdala' & & $35.05,<0.0001$ & $1.01,0.96-1.07$ & $0.84,0.76-0.93$ & $1.39,1.21-1.61$ & $0.96,0.87-1.07$ \\
\hline Hippocampus $^{1}$ & & $38.73,<0.0001$ & $0.93,0.88-0.97$ & $0.97,0.89-1.06$ & $1.43,1.25-1.63$ & $0.96,0.84-1.10$ \\
\hline Left dorsal striatum² & & $32.97,<0.0001$ & $0.88,0.81-0.96$ & $0.80,0.68-0.93$ & $1.72,1.36-2.19$ & $0.99,0.67-1.48$ \\
\hline Right dorsal striatum ${ }^{2}$ & & $25.32,<0.0001$ & $0.87,0.80-0.95$ & $0.80,0.68-0.92$ & $1.60,1.24-2.05$ & $1.00,0.70-1.43$ \\
\hline Ventral striatum ${ }^{2}$ & $14.86,<0.0001$ & & $0.63,0.50-0.80$ & $0.63,0.50-0.80$ & $0.63,0.50-0.80$ & $0.63,0.50-0.80$ \\
\hline Left insula & & $12.79,0.005$ & $1.05,0.75-1.46$ & $1.41,0.65-3.04$ & $6.71,2.53-17.84$ & $1.40,0.38-5.19$ \\
\hline Right insula & $7.52,0.006$ & & $0.70,0.53-0.90$ & $0.70,0.53-0.90$ & $0.70,0.53-0.90$ & $0.70,0.53-0.90$ \\
\hline
\end{tabular}

$D x$ diagnosis, $A S D$ autism spectrum disorder, $A D H D$ attention-deficit/hyperactivity disorder, OCD obsessive-compulsive disorder, SCQ Social Communication Questionnaire, social interaction, and communication items only, OR odds ratios, FDR false discovery rate

ORs are the odds of scoring on an SCQ item per mm increase in thickness, or ${ }^{1} 0.1 \mathrm{~cm}^{3}$ increase in volume, or ${ }^{2} 1.0 \mathrm{~cm}$ increase in volume. Models treat SCQ scored/total as the dependent variable, and have been adjusted for the effects of age, sex, diagnosis, scanner upgrade, and diagnosis-by-region interactions, as well as whole-brain volume for volumetric structures. Where diagnosis-by-region interactions were non-significant $(p>0.05)$, they were dropped from the model. Bolded values remained significant $(q<0.05)$ after FDR correction

(Fig. 2, bottom panels). For the ADHD group, thicker cortices were associated with greater social deficits in the right temporal lobe, and the left occipital lobe. No regions remained significant after FDR correction for the OCD or control groups on the RMET.

\section{The association between brain structure and social communication abilities may vary by age}

As an exploratory analysis, we also examined for age effects on the association between brain region and social deficits by checking for age-by-region interaction terms for the regions of interest. On the SCQ, the right lateral region showed a highly significant $\left(\chi^{2}=21.82, p<0.0001\right)$ age-by-region interaction. Specifically, the association between right lateral region thickness and SCQ score was more pronounced at younger ages (age 7 OR: 0.17 $(0.10-0.30)$ ), and was less significant in older children (age 11 OR: 0.39 (0.29-0.53), age 15 OR: 0.89 (0.57-1.35)). Several other regions showed a similar pattern, although the effect was less pronounced and would become nonsignificant after a correction for multiple comparisons (age-by-region interaction term $p$ value for left lateral regions $=0.03$, frontal regions $=0.02$, hippocampus $=$ 0.02 , right dorsal striatum $=0.01$ ). There were no significant age-by-region interactions for analyses involving the RMET. For the right lateral region, we then also tested for a three-way interaction term "age-by-region-by-diagnosis," to determine whether the age effects on the brain-behavior relationship varied across diagnostic groups. There was a nominally significant three-way interaction term $(p=0.05)$. ASD and ADHD had more significant associations between brain and behavior on the SCQ at younger ages (OR age 7 for ASD: 0.20 (0.10-0.40); OR age 7 ADHD: 0.24 (0.08-0.70); OR age 15 ASD: 1.81 (1.07-3.06); OR age 15 ADHD: 0.94 (0.23-3.82)), whereas the opposite pattern was observed for the OCD and control groups (OR age 7 control: 0.24 (0.03-1.80), OR age 7 OCD: 0.50 (0.02-14.2); OR age 15 control: 0.02 (0.01-0.4), OR age 15 OCD (0.12 (0.03-0.45)).

\section{Exploratory analysis involving oxytocin receptor (OXTR) polymorphisms}

There were 100 participants with ASD, and 51 participants with ADHD who also had genotype data available regarding single-nucleotide polymorphisms (SNPs) in the OXTR gene (Supplementary Table 7). Previous work on the same study sample suggested three SNPs in OXTR ( $r s 53576, r s 237997$, and rs2254298) modify the severity of social deficits in ASD and ADHD ${ }^{72}$. We therefore examined for associations between $O X T R$ genotype and brain 


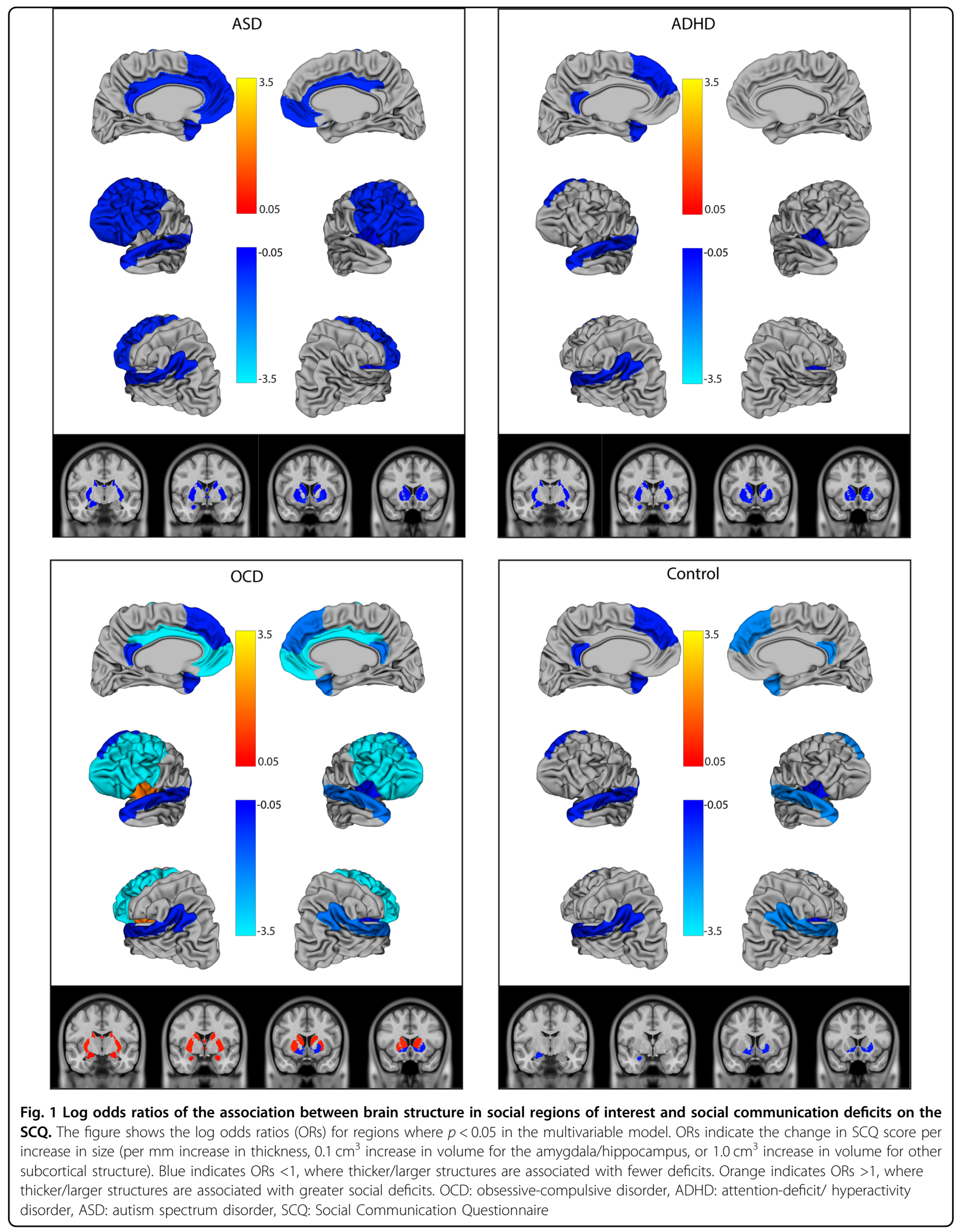


Table 3 Association between social regions of interest and social deficits on the RMET

\begin{tabular}{|c|c|c|c|c|c|c|}
\hline \multirow[t]{2}{*}{ Region } & \multicolumn{2}{|l|}{ Wald $X^{2}, p$ value } & \multirow[t]{2}{*}{ OR ASD } & \multirow[t]{2}{*}{ OR ADHD } & \multirow[t]{2}{*}{ OR OCD } & \multirow[t]{2}{*}{ OR Control } \\
\hline & Region & Region $\times \mathrm{Dx}$ & & & & \\
\hline \multicolumn{7}{|c|}{ Lateral mentalization regions } \\
\hline Left lateral regions & & $8.16, p=0.04$ & $0.60,0.36-0.98$ & $1.34,0.73-2.46$ & $2.77,0.72-10.61$ & $1.42,0.50-4.12$ \\
\hline Right lateral regions & & $9.51, p=0.02$ & $0.55,0.35-0.86$ & $1.52,0.86-2.68$ & $1.24,0.60-2.55$ & $0.94,0.34-2.65$ \\
\hline \multicolumn{7}{|l|}{ Frontal cognitive regions } \\
\hline Frontal regions & $0.58, p=0.4$ & & $0.85,0.55-1.30$ & $0.85,0.55-1.30$ & $0.85,0.55-1.30$ & $0.85,0.55-1.30$ \\
\hline \multicolumn{7}{|c|}{ Deeper and subcortical affective regions } \\
\hline Left amygdala ${ }^{1}$ & 23.34, $\boldsymbol{p}<0.0001$ & & $0.88,0.83-0.92$ & $0.88,0.83-0.92$ & $0.88,0.83-0.92$ & $0.88,0.83-0.92$ \\
\hline Right amygdala' & $8.74, p=0.003$ & & $0.94,0.90-0.98$ & $0.94,0.90-0.98$ & $0.94,0.90-0.98$ & $0.94,0.90-0.98$ \\
\hline Hippocampus $^{1}$ & $10.71, p=0.001$ & & $0.93,0.89-0.97$ & $0.93,0.89-0.97$ & $0.93,0.89-0.97$ & $0.93,0.89-0.97$ \\
\hline Left dorsal striatum² & $0.05, p=0.8$ & & $0.99,0.92-1.07$ & $0.99,0.92-1.07$ & $0.99,0.92-1.07$ & $0.99,0.92-1.07$ \\
\hline Right dorsal striatum ${ }^{2}$ & $0.00, p=0.9$ & & $1.00,0.92-1.08$ & $1.00,0.92-1.08$ & $1.00,0.92-1.08$ & $1.00,0.92-1.08$ \\
\hline Ventral striatum $^{2}$ & $0.47, p=0.5$ & & $1.09,0.86-1.38$ & $1.09,0.86-1.38$ & $1.09,0.86-1.38$ & $1.09,0.86-1.38$ \\
\hline Left insula & $0.58, p=0.4$ & & $0.89,0.65-1.21$ & $0.89,0.65-1.21$ & $0.89,0.65-1.21$ & $0.89,0.65-1.21$ \\
\hline Right insula & & $9.63, p=0.02$ & $0.48,0.33-0.69$ & $1.21,0.72-2.03$ & $1.02,0.48-2.17$ & $0.61,0.28-1.31$ \\
\hline
\end{tabular}

$D x$ diagnosis, $A S D$ autism spectrum disorder, $A D H D$-attention-deficit/hyperactivity disorder, $O C D$ obsessive-compulsive disorder, $R M E T$ Reading the Mind in the Eyes Test incorrect item, $O R$ odds ratios

ORs are the odds of scoring incorrectly on an RMET item per $\mathrm{mm}$ increase in thickness, or ${ }^{1} 0.1 \mathrm{~cm}^{3}$ increase in volume, or ${ }^{2} 1.0 \mathrm{~cm}$ increase in volume. Models treat RMET incorrect/total as the dependent variable, and have been adjusted for the effects of age, sex, diagnosis, scanner upgrade, and diagnosis-by-region interactions, as well as whole-brain volume for volumetric structures. Where diagnosis-by-region interactions were non-significant $(p>0.05)$, they were dropped from the model. Bolded values remained significant $(q<0.05)$ after FDR correction

structure in brain regions previously found to be affected by $O X T R$ genotype in control populations (insula, limbic system, dorsal cingulate, temporal-parietal (lateral) regions) ${ }^{73-80}$. Exploratory analyses (described in Supplementary Methods) failed to detect any significant associations between $O X T R$ genotype and brain structure after correction for multiple comparisons (Bonferroni correction with 24 tests, $p=0.002$ ), with the exception of the left insula, in ADHD (Supplementary Table 8). Here, rs53576 AA allele carriers had thicker cortices in the insula compared to other genotype groups (AA vs. GA $p=$ 0.0005 , AA vs. GG $p=0.0008)$.

\section{Discussion}

In this study, we examined and compared the structural neuroimaging correlates of social abilities across a large sample of children and youth with different neurodevelopmental disorders. Overall data suggest similarities in the biological substrates of social communication abilities in ASD and ADHD; both had widely distributed associations between brain structure and SCQ score, involving multiple subcortical and cortical regions. Exploratory analyses identified age-associated difference in this effect in the right lateral regions, which were congruent in ASD and ADHD as well. For social perception on the RMET, all four groups showed a similar pattern of associations involving subcortical regions, while participants with ASD also had significant findings in the right insula and the right lateral regions. Across all diagnostic groups, associations were highly heterogeneous.

Our results are consistent with emerging data suggesting ASD and ADHD may be overlapping conditions, with related neuropathology and symptomatology ${ }^{81,82}$. For example, diffusion tensor imaging has shown a lack of diagnostic differences in fractional anisotropy of white matter tracts between ASD and ADHD, while OCD appeared distinct ${ }^{8}$. Latent class analysis of symptom surveys and cognitive profiles suggest overlapping cognitive deficits in both disorders ${ }^{81}$. Patterns of social perception deficits were strikingly similar in ASD and ADHD as well $^{1}$. Rare copy number variants affecting many of the same genes have been shown to contribute to risk for both ASD and $\mathrm{ADHD}^{83}$.

Previous studies examining the association between brain structure and social deficits have yielded inconsistent results. Most studies have used the Social Responsiveness Scale (SRS) or subscales of the ADI-R to quantify social communication deficits, primarily in control populations. Recent work suggests the SRS may be highly sensitive to non-specific behavioral problems, 


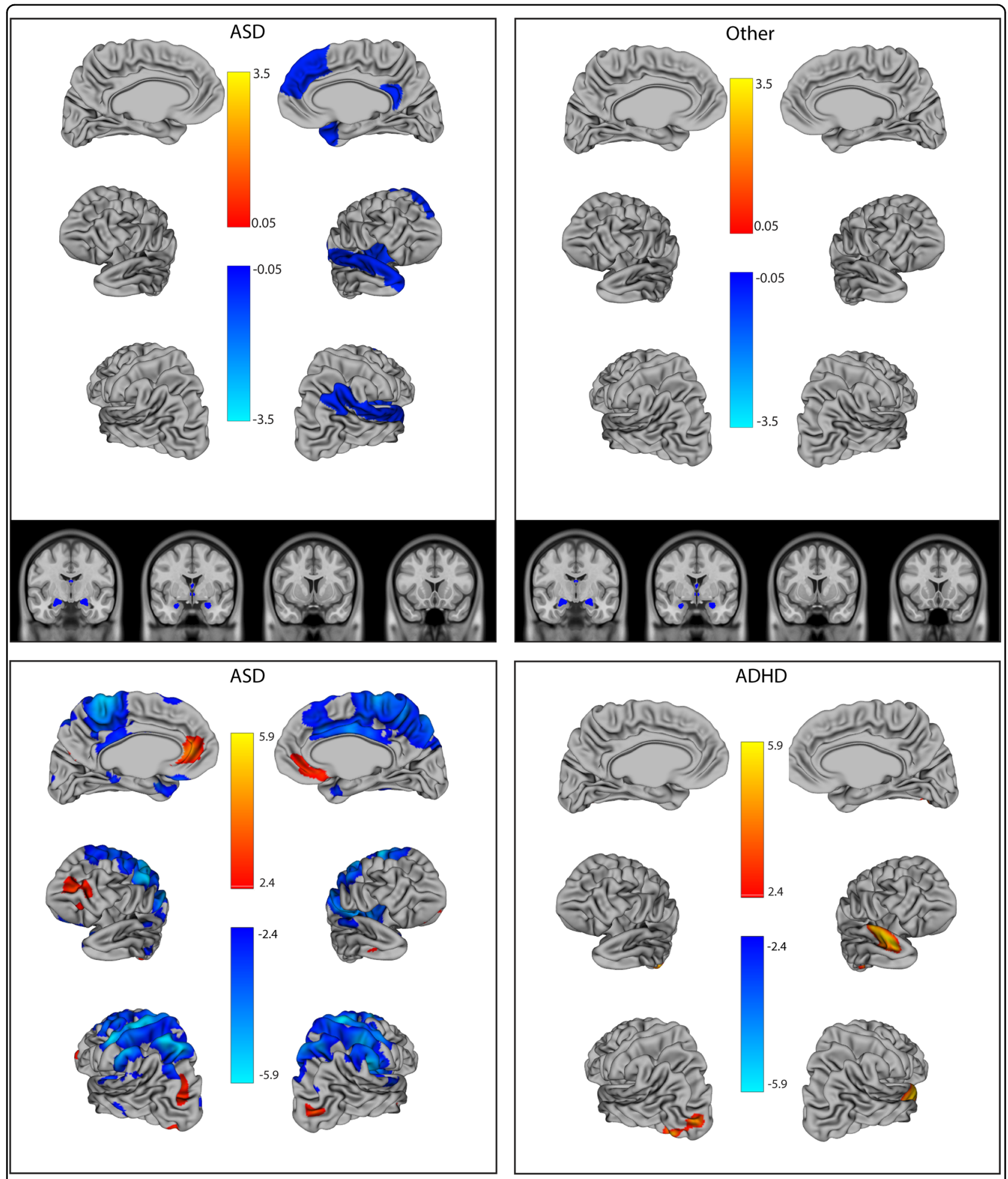

Fig. 2 Log odds ratios of the association between brain structure and social perception deficits on the RMET. Top panels show the region of interest approach; log odds ratios (ORs) are for regions that were significant the multivariable model after false discovery rate (FDR) correction ( $q=$ 0.05). The bottom panels present the vertex-wise analysis for ASD and ADHD; vertices are shown that remained significant after an FDR correction at $q=0.05$ (no vertices remained significant for the controls or OCD group). ORs indicate the change in RMET score per increase in size (per mm increase in thickness, $0.1 \mathrm{~cm}^{3}$ increase in volume for the amygdala/hippocampus, or $1.0 \mathrm{~cm}^{3}$ increase in volume for other subcortical structures). Blue indicates ORs $<1$, where thicker/larger structures are associated with fewer deficits. Orange indicates ORs $>1$, where thicker/larger structures are associated with greater social deficits. OCD: obsessive-compulsive disorder, ADHD: attention-deficit/ hyperactivity disorder, ASD: autism spectrum disorder, RMET: Reading the Mind in the Eyes Test 

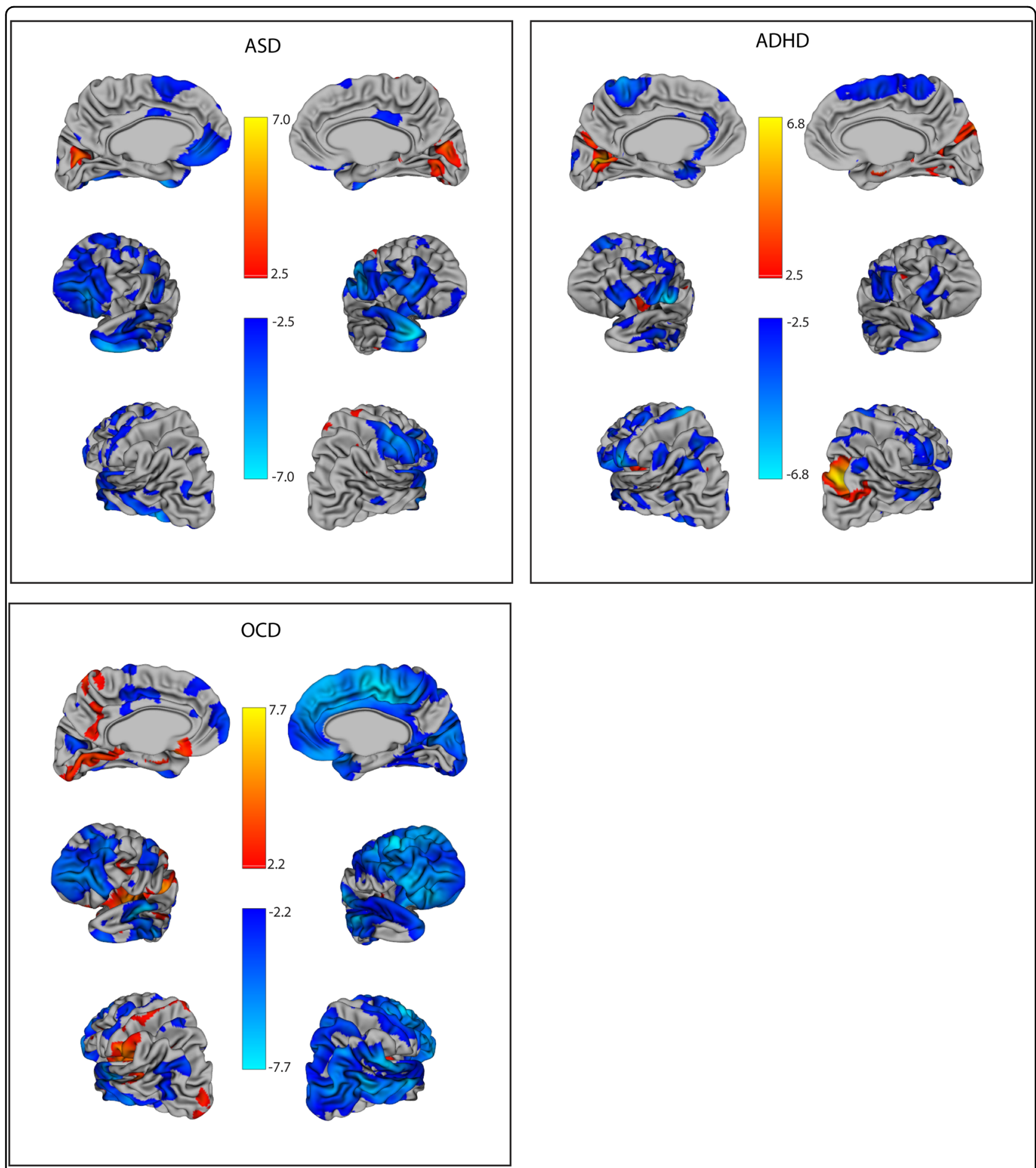

Fig. 3 Log odds ratios of the association between cortical thickness and social communication deficits on the SCQ using a vertex-wise analysis. Vertices are shown that remained significant after an false discovery rate (FDR) correction at $q=0.05$ (no vertices remained significant for the control group). Odds ratios (ORs) indicate the change in SCQ score per increase in size (per mm increase in thickness, $0.1 \mathrm{~cm}^{3}$ increase in volume for the amygdala/hippocampus, or $1.0 \mathrm{~cm}^{3}$ increase in volume for other subcortical structures). Blue indicates ORs $<1$, where thicker/larger structures are associated with fewer deficits. Orange indicates ORs $>1$, where thicker/larger structures are associated with greater social deficits. OCD: obsessivecompulsive disorder, ADHD: attention-deficit/hyperactivity disorder, ASD: autism spectrum disorder, SCQ: Social Communication Questionnaire 
reflecting general levels of impairment more so than social deficits per $\mathrm{se}^{84}$. In this study, we used the SCQ, which has been found to be a better screening instrument for ASD compared to the SRS ${ }^{85}$, and is not affected by IQ like other measures ${ }^{85,86}$. Despite different measures, our results using the SCQ (i.e., thicker cortices in frontal/ temporal-parietal regions being associated with fewer deficits) are broadly consistent with several studies examining structural neuroimaging correlates of the SRS/ ADI-R in control participants ${ }^{87-89}$ and in ASD $^{26,90}$. The direction of our results are inconsistent with two larger studies, the first using the SRS in a large population of typically developing in 6-10 year olds ${ }^{24}$, and the second using the Autism Diagnostic Observation Schedule in ASD across the lifespan ${ }^{29}$. Difference in results may be explained in part by true biological differences in the neural substrates of these measures, heterogeneity across disorders and populations, variability in the age ranges of included participants, use of models that may or may not account for comorbid symptomatology/differences in IQ, and variation in the measurement aspects of the underlying social construct in different disorders.

Functional and structural neuroimaging work has associated RMET performance with activation/size of the inferior frontal gyrus, middle/posterior temporal regions, and the amygdala/hippocampus in typically developing adults $^{91-94}$, although findings may defer in $\mathrm{ASD}^{67,95}$. Our results for the RMET highlight subcortical structures as being important across disorders; children with ASD also had associations in the lateral mentalization regions and in the insula. It is possible that this finding is reflective of more immature or inefficient social perception abilities in children with ASD, leading them to rely on additional cortical circuits to make social evaluations ${ }^{96}$.

Exploratory analyses identified age-associated differences in the association between right lateral region thickness and SCQ score; data also suggested that these age-associated differences may vary by diagnostic group. Existing literature supports the hypothesis that the neural substrates of social abilities may change over the course of development. For example, previous longitudinal work has shown that the patterns of functional MRI (fMRI) activation during facial/emotion recognition tasks change with age, and in particular, after puberty ${ }^{97,98}$. In control participants, completion of the RMET was associated with activation in the posterior superior temporal sulcus across age ranges, but younger children (with potentially more immature social perception abilities) also activated the prefrontal cortex, the inferior frontal gyrus, and the temporal pole during this task as well ${ }^{96}$. Given that the direction of the association between cortical thickness and general intelligence may change over childhood (from a negative correlation in younger children to a positive correlation in older (hildren $)^{99}$, and that neurodevelopmental disorders such as ASD and ADHD are associated with aberrant cortical maturation ${ }^{15,18}$, further research is merited in order to characterize disorderspecific longitudinal changes in the neural substrates of social deficits.

The pattern involving OCD on the SCQ is notably different with larger subcortical structures being associated with greater social impairments. Previous research has found frontal-subcortical hyperactivity during processing of emotional stimuli in OCD on $\mathrm{PMRI}^{100}$. Therefore, it is possible that the social deficits associated with increased subcortical volumes reflect aberrant processing of social-emotional information in OCD. Consistent with this hypothesis, the distinct OCD pattern in our data persisted even after adjusting for severity of OCD symptoms. The OCD group also showed strong lateralization effects with respect to the association between social abilities and insula thickness, consistent with previous data in control populations ${ }^{70}$. Overall, despite overlapping symptomatology with other disorders (in particular, repetitive behavior in ASD), the pattern of brain-behavior associations involving the OCD group appeared quite distinct.

The structural finding of nominally larger hippocampal volumes in OCD is consistent with data from a recent large meta-analysis showing a trend towards larger hippocampi $(p=0.09)$ in 107 pediatric OCD patients compared with 210 controls $^{21}$. This stands in contrast to adult populations, where smaller hippocampi were found ${ }^{21}$. Notably, across other social brain regions of interest, there were no significant structural differences in thickness or volume between the four diagnostic groups. Metaanalyses comparing over 1000 participants with $\mathrm{ASD}^{20}$, or $\mathrm{ADHD}^{23}$, to control participants have found smaller subcortical volumes in both ASD and ADHD. Cortical differences have varied by study, and by age, for both disorders, however ${ }^{18,20}$. These results further highlight a lack of biological differences between ASD and ADHD in spite of differences in mean scores on the SCQ and RMET.

Oxytocin is a pituitary neuropeptide known to affect social behavior. A few small studies, in typically developing adult cohorts, have examined whether common genetic differences in the oxytocin receptor gene (OXTR) corresponded with differences in structural neuroanatomy ${ }^{77-80,101}$. Specifically, different $O X T R$ rs 2254298 and rs53576 genotypes corresponded with differences in amygdala volumes ${ }^{77,78,80}$, and OXTR rs2254298 genotype was associated with differences in thickness of the right insula and dorsal anterior cingulate as well ${ }^{79,101}$. Our exploratory analyses did not reproduce these findings in children with neurodevelopmental disorders, although rs53576 genotype was associated with thickness in the left insula in the ADHD group. Despite this, overall trends 
suggested a similar direction of gene-brain associations in ASD and ADHD.

In terms of limitations, sample sizes were unequal between groups, contributing to less confidence to detect differences in the OCD and control groups. Regions were grouped anatomically and functionally to facilitate description of overall patterns; consensus has yet to be reached with the respect to the specific regions and functions of social neural networks. The SCQ is a parent report measure that captures complex aspects of social communication (making friends, using non-verbal communication, interest in others); diagnostic differences in the measurement aspects of this measure were not the focus of the paper but could also contribute to results. SCQ scores were right skewed in controls, ADHD, and OCD, but left skewed in ASD, raising the possibility of influential outliers driving results. Case-wise diagnostics were conducted and repeat analyses excluding 13 potential influential outliers based on difchi-squared values yielded the same pattern of findings (note no dfbetas were $>2$ ). Restricting the range of SCQ scores to 0-15 resulted in similar patterns as well, although less significant results. The group with ASD tended to be higher functioning; results may be less generalizable to a more severely affected population. Also the oxytocin results should be considered exploratory in view of the small sample size and the multiple testing.

Overall, this study revealed that the structural neuroimaging correlates of social communication and social perception deficits vary across neurodevelopmental disorders. There were more similarities than differences between ASD and ADHD, even on an ASD-specific outcome measure. Data support a common underlying biology and the blurring of the diagnostic boundaries between these two disorders.

\section{Acknowledgements}

This research was supported by the grant IDS-I I-02 from the Ontario Brain Institute. We thank the following individuals for research support and data collection: Tara Goodale, M.Sc., Reva Schachter, M.Sc., Mithula Sriskandarajah, B. Sc., Marlena Colasanto, M.Sc., Jennifer Gomez, M.A., and Laura Park, M.Sc, from The Hospital for Sick Children; Susan Day Fragiadakis, M.A., Naomi Peleg, M.Sc., and Leanne Ristic, B.A., from Holland Bloorview; Richa Mehta, B.A., Christina Sommerdyk, M.Sc., from the Lawson Health Research Institute; Carolyn Russell, B.Sc., Alessia Greco, M.A., Mike Chalupka, B.A., B.Sc., Christina Chrysler, B.A., Irene O'Connor, M.Ed. Psych., from McMaster Children's Hospital. P.D.A. holds the Alberta Innovates Translational Health Chair in Child and Youth Mental Health . P.Z. holds the Patsie and Jamie Anderson Chair in Child and Youth Mental Health. D.A.B was supported by the American Psychiatric Association Resident Psychiatric Research Scholars Program, the American Academy of Child and Adolescent Psychiatry Pilot Research Award, the University of Toronto Clinician Scientist Program, and the University of Toronto Postgraduate Research Awards.

\footnotetext{
Author details

${ }^{1}$ Department of Psychiatry, University of Toronto, Toronto, ON, Canada. ${ }^{2}$ Clinical Research Services, The Hospital for Sick Children, and the Dalla Lana School of Public Health, University of Toronto, Toronto, ON, Canada. ${ }^{3}$ The Centre for Applied Genomics, The Hospital for Sick Children, Toronto, ON, Canada. ${ }^{4}$ Mouse Imaging Centre, The Hospital for Sick Children, Toronto, ON,
}

Canada. ${ }^{5}$ The McLaughlin Centre and the Department of Molecular Genetics, University of Toronto, Toronto, ON, Canada. ${ }^{6}$ Department of Psychiatry, Neurosciences and Mental Health, The Hospital for Sick Children, Toronto, ON, Canada. ${ }^{7}$ Mathison Centre for Mental Health Research \& Education, Hotchkiss Brain Institute, Cumming School of Medicine and Department of Psychiatry and Medical Genetics, University of Calgary, Calgary, AL, Canada. ${ }^{8}$ The Centre for Addiction and Mental Health, Toronto, ON, Canada. ${ }^{9}$ Department of Psychiatry, Western University and Children's Health Research Institute, London, ON, Canada. ${ }^{10}$ Department of Psychiatry and Behavioural Neurosciences, McMaster University, Hamilton, ON, Canada. ${ }^{11}$ Department of Paediatrics, University of Toronto, Toronto, ON, Canada. ${ }^{12}$ Autism Research Centre, Bloorview Research Institute, Holland Bloorview Kids Rehabilitation Hospital, Toronto, ON, Canada. ${ }^{13}$ University of Toronto, Institute of Biomaterial and Biomedical Engineering, Toronto, ON, Canada. ${ }^{14}$ Department of Medical Biophysics, University of Toronto, Toronto, ON, Canada

\section{Competing interests}

R.J.S. has consulted to Highland Therapeutics, Eli Lilly and Co., and Purdue Pharma. He has commercial interest in a cognitive rehabilitation software company, "eHave." P.D.A. holds a patent for "SLCIAI Marker for Anxiety

Disorder" granted 6 May 2008. P.S. has received royalties from Guilford Press. E. A. has served as a consultant to Roche. She has received grant funding from SanofiCanada and SynapDx. She has received royalties from APPI and Springer. E.A. and A.K. hold a provisional patent for the device, "Anxiety Meter." S.W.S. is an academic scientific consultant advising or is in licensing arrangements with Athena, Diagnostics, King Abdulaziz University Lineagen, Deep Genomics and Population Diagnostics. He also holds the CIHR-GlaxoSmithKline Endowed Chair in Genome Sciences at the Hospital for Sick Children and University of Toronto. The remaining authors declare no competing interests.

\section{Publisher's note}

Springer Nature remains neutral with regard to jurisdictional claims in published maps and institutional affiliations.

Supplementary information accompanies this paper at (https://doi.org/ 10.1038/s41398-019-0382-0).

Received: 27 July 2018 Revised: 21 November 2018 Accepted: 1 January 2019

Published online: 04 February 2019

\section{References}

1. Baribeau, D. A. et al. Examining and comparing social perception abilities across childhood-onset neurodevelopmental disorders. J. Am. Acad. Child Adolesc. Psychiatry 54, 479-486 e1 (2015).

2. Liu, W. et al. Disassociation of cognitive and affective aspects of theory of mind in obsessive-compulsive disorder. Psychiatry Res. 255, 367-372 (2017).

3. Kang, J. I., Namkoong, K., Yoo, S. W., Jhung, K. \& Kim, S. J. Abnormalities of emotional awareness and perception in patients with obsessive-compulsive disorder. J. Affect Disord. 141, 286-293 (2012).

4. Cullen, B. et al. Social and communication difficulties and obsessivecompulsive disorder. Psychopathology 41, 194-200 (2008).

5. Kim, K, Reynolds, K. \& Alfano, C. Social impairment in children with obsessive compulsive disorder: Do comorbid problems of inattention and hyperactivity matter? J. Obsessive Compuls. Relat. Disord. 1, 228-233 (2012).

6. Lionel, A. C. et al. Disruption of the ASTN2/TRIM32 locus at 9q33.1 is a risk factor in males for autism spectrum disorders, ADHD and other neurodevelopmental phenotypes. Hum. Mol. Genet. 23, 2752-2768 (2014).

7. van der Plas, E., Dupuis, A., Arnold, P., Crosbie, J. \& Schachar, R. Association of autism spectrum disorder with obsessive-compulsive and attention-deficit/ hyperactivity traits and response inhibition in a community sample. J. Autism Dev. Disord. 46, 3115-3125 (2016).

8. Ameis, S. H. et al. A diffusion tensor imaging study in children with adhd autism spectrum disorder, OCD, and matched controls: distinct and nondistinct white matter disruption and dimensional brain-behavior relationships. Am. J. Psychiatry appiajp201615111435 (2016). 
9. Insel, T. et al. Research domain criteria (RDoC): toward a new classification framework for research on mental disorders. Am. J. Psychiatry 167, 748-751 (2010).

10. Alcala-Lopez, D. et al. Computing the social brain connectome across systems and states. Cereb. Cortex 1-26 (2017).

11. Van Overwalle, F. Social cognition and the brain: a meta-analysis. Hum. Brain Mapp. 30, 829-858 (2009).

12. Stanley, D. A. \& Adolphs, R. Toward a neural basis for social behavior. Neuron 80, 816-826 (2013)

13. Kennedy, D. P. \& Adolphs, R. The social brain in psychiatric and neurological disorders. Trends Cogn. Sci. 16, 559-572 (2012).

14. Reeck, C., Ames, D. R. \& Ochsner, K. N. The social regulation of emotion: an integrative, cross-disciplinary model. Trends Cogn. Sci. 20, 47-63 (2016).

15. Zielinski, B. A. et al. Longitudinal changes in cortical thickness in autism and typical development. Brain 137(Part 6), 1799-1812 (2014).

16. Doyle-Thomas, K. A. et al. Effects of age and symptomatology on cortical thickness in autism spectrum disorders. Res. Autism Spectr. Disord. 7, 141-150 (2013).

17. DeRamus, T. P. \& Kana, R. K. Anatomical likelihood estimation meta-analysis of grey and white matter anomalies in autism spectrum disorders. Neuroimage Clin. 7, 525-536 (2015).

18. Shaw, P. et al. Attention-deficit/hyperactivity disorder is characterized by a delay in cortical maturation. Proc. Natl. Acad. Sci. USA 104, 19649-19654 (2007).

19. Almeida Montes, L. G. et al. Brain cortical thickness in ADHD: age, sex, and clinical correlations. J. Atten. Disord. 17, 641-654 (2013).

20. van Rooij, D. et al. Cortical and subcortical brain morphometry differences between patients with autism spectrum disorder and healthy individuals across the lifespan: results from the enigma ASD Working Group. Am. J. Psychiatry appiajp201717010100 (2017).

21. Boedhoe, P. S. et al. Distinct subcortical volume alterations in pediatric and adult OCD:a worldwide meta- and mega-analysis.Am. J. Psychiatry 174, 60-69 (2017).

22. Boedhoe, P. S. W. et al. Cortical abnormalities associated with pediatric and adult obsessive-compulsive disorder: findings from the ENIGMA ObsessiveCompulsive Disorder Working Group. Am. J. Psychiatry 175, 453-462 (2018).

23. Hoogman, M. et al. Subcortical brain volume differences in participants with attention deficit hyperactivity disorder in children and adults: a crosssectional mega-analysis. Lancet Psychiatry 4, 310-319 (2017).

24. Blanken, L. M. et al. Cortical morphology in 6- to 10-year old children with autistic traits: a population-based neuroimaging study. Am. J. Psychiatry 172 479-486 (2015).

25. Hadjikhani, N., Joseph, R. M., Snyder, J. \& Tager-Flusberg, H. Anatomical differences in the mirror neuron system and social cognition network in autism. Cereb. Cortex 16, 1276-1282 (2006).

26. Wallace, G. L. et al. Longitudinal cortical development during adolescence and young adulthood in autism spectrum disorder: increased cortical thinning but comparable surface area changes. J. Am. Acad. Child Adolesc. Psychiatry 54, 464-469 (2015).

27. Richter, J. et al. Reduced cortical thickness and its association with socia reactivity in children with autism spectrum disorder. Psychiatry Res. 234 15-24 (2015).

28. Hardan, A. Y., Libove, R. A., Keshavan, M. S., Melhem, N. M. \& Minshew, N. J. A preliminary longitudinal magnetic resonance imaging study of brain volume and cortical thickness in autism. Biol. Psychiatry 66, 320-326 (2009).

29. Khundrakpam, B. S., Lewis, J. D., Kostopoulos, P., Carbonell, F. \& Evans, A. C. Cortical thickness abnormalities in autism spectrum disorders through late childhood, adolescence, and adulthood: a large-scale MRI study. Cereb. Cortex 27, 1721-1731 (2017)

30. Ecker, C. et al. Brain surface anatomy in adults with autism: the relationship between surface area, cortical thickness, and autistic symptoms. JAMA Psychiatry 70, 59-70 (2013).

31. Hotier, S. et al. Social cognition in autism is associated with the neurodevelopment of the posterior superior temporal sulcus. Acta Psychiatr. Scand. 136, 517-525 (2017).

32. O'Dwyer, L. et al. Brain volumetric correlates of autism spectrum disorder symptoms in attention deficit/hyperactivity disorder. PLOS ONE 9, e101130 (2014).

33. O'Dwyer, L. et al. Decreased left caudate volume is associated with increased severity of autistic-like symptoms in a cohort of ADHD patients and their unaffected siblings. PLOS ONE 11, e0165620 (2016).
34. Panizzon, M. S. et al. Distinct genetic influences on cortical surface area and cortical thickness. Cereb. Cortex 19, 2728-2735 (2009).

35. Schnack, H. G. et al. Changes in thickness and surface area of the human cortex and their relationship with intelligence. Cereb. Cortex 25, 1608-1617 (2015).

36. Wierenga, L. M., Langen, M., Oranje, B. \& Durston, S. Unique developmental trajectories of cortical thickness and surface area. Neuroimage $\mathbf{8 7}, 120-126$ (2014).

37. Schumann, C. M. \& Nordahl, C. W. Bridging the gap between MRI and postmortem research in autism. Brain Res. 1380, 175-186 (2011).

38. Frith, U. Mind blindness and the brain in autism. Neuron 32, 969-979 (2001).

39. Willcutt, E. G., Doyle, A. E., Nigg, J. T., Faraone, S. V. \& Pennington, B. F. Validity of the executive function theory of attention-deficit/hyperactivity disorder: a meta-analytic review. Biol. Psychiatry 57, 1336-1346 (2005).

40. Figee, M. et al. Dysfunctional reward circuitry in obsessive-compulsive disorder. Biol. Psychiatry 69, 867-874 (2011).

41. Lord, C. et al. The autism diagnostic observation schedule-generic: a standard measure of social and communication deficits associated with the spectrum of autism. J. Autism Dev. Disord. 30, 205-223 (2000).

42. Lord, C., Rutter, M. \& Le Couteur, A. Autism Diagnostic Interview-Revised: a revised version of a diagnostic interview for caregivers of individuals with possible pervasive developmental disorders. J. Autism Dev. Disord. 24, 659-685 (1994).

43. Kaufman, J. et al. Schedule for Affective Disorders and Schizophrenia for School-Age Children-Present and Lifetime Version (K-SADS-PL): initial reliability and validity data. J. Am. Acad. Child Adolesc. Psychiatry 36, 980-988 (1997).

44. Ickowicz, A. et al. The parent interview for child symptoms: a situationspecific clinical research interview for attention-deficit hyperactivity and related disorders. Can. J. Psychiatry 51, 325-328 (2006).

45. Scahill, L. et al. Children's Yale-Brown Obsessive Compulsive Scale: reliability and validity. J. Am. Acad. Child Adolesc. Psychiatry 36, 844-852 (1997).

46. Berument, S. K., Rutter, M., Lord, C., Pickles, A. \& Bailey, A. Autism screening questionnaire: diagnostic validity. Br. J. Psychiatry 175, 444-451 (1999).

47. Baron-Cohen, S., Wheelwright, S., Hill, J., Raste, Y. \& Plumb, I. The "Reading the Mind in the Eyes" Test revised version: a study with normal adults, and adults with Asperger syndrome or high-functioning autism. J. Child Psychol. Psychiatry 42, 241-251 (2001).

48. Baron-Cohen, S., Wheelwright, S., Spong, A., Scahill, V. \& Lawson, J. Are intuitive physics and intuitive psychology independent? A test with children with Asperger syndrome. J. Dev. Learn. Disord. 5, 47-78 (2001).

49. Achenbach, T. M., Vermont, VDoPUo, Edelbrock, CS. Manual for the Child Behavior Checklist and Revised Child Behavior Profile (Department of Psychiatry of the University of Vermont, Burlington, 1983).

50. Park, L. S. et al. The Toronto Obsessive-Compulsive Scale: psychometrics of a dimensional measure of obsessive-compulsive traits. J. Am. Acad. Child Adolesc. Psychiatry 55, 310-318 e4 (2016).

51. Ad-Dab'bagh, Y. L. O. et al. The CIVET Image-Processing Environment: a fully automated comprehensive pipeline for anatomical neuroimaging research In Proc. 12th Annual Meeting of the Organization for Human Brain Mapping (eds. Corbetta M., Nichols T., Pietrini P.) 31, Supp1, 1-242 (Florence, Italy, 2006).

52. Sled, J. G., Zijdenbos, A. P. \& Evans, A. C. A nonparametric method for automatic correction of intensity nonuniformity in MRI data. IEEE Trans. Med. Imaging 17, 87-97 (1998).

53. Grabner, G. et al. Symmetric atlasing and model based segmentation: an application to the hippocampus in older adults. adults. Medical image computing and computer-assistedintervention: MICCAl. Int. Conf. Med. Image Comput. Comput.-Assist. Interv. 9(Part 2), 58-66 (2006).

54. Collins, D. L., Neelin, P., Peters, T. M. \& Evans, A. C. Automatic 3D inter-subject registration of MR volumetric data in standardized Talairach space. J. Comput. Assist. Tomogr. 18, 192-205 (1994)

55. Smith, S. M. Fast robust automated brain extraction. Hum. Brain Mapp. 17, 143-155 (2002).

56. Tohka, J., Zijdenbos, A. \& Evans, A. Fast and robust parameter estimation for statistical partial volume models in brain MRl. Neuroimage 23, 84-97 (2004).

57. Zijdenbos, A., Forghani, R. \& Evans, A. in Medical Image Computing and Computer-Assisted Interventation (MICCAl98) (eds Wells, W. M., Colchester, A. \& Delp, S.) 439-448 (Springer, Cambridge, MA, and Berlin, Heidelberg, 1998). 
58. Kim, J. S. et al. Automated 3-D extraction and evaluation of the inner and outer cortical surfaces using a Laplacian map and partial volume effect classification. Neuroimage 27, 210-221 (2005).

59. MacDonald, D., Kabani, N., Avis, D. \& Evans, A. C. Automated 3-D extraction of inner and outer surfaces of cerebral cortex from MRI. Neuroimage 12, 340-356 (2000).

60. Chung, M. K. \& Taylor J. Diffusion smoothing on brain surface via finite element method. IEEE Int. Symp. Biomed. Imag. 562, 432-435 (2004).

61. Robbins, S. M. Anatomical Standardization of the Human Brain in Euclidean 3Space and on the Cortical 2-Manifold (McGill University, Montreal, 2004).

62. Lyttelton, O., Boucher, M., Robbins, S. \& Evans, A. An unbiased iterative group registration template for cortical surface analysis. Neuroimage 34, 1535-1544 (2007).

63. Boucher, M., Whitesides, S. \& Evans, A. Depth potential function for folding pattern representation, registration and analysis. Med. Image Anal. 13, 203-214 (2009).

64. Lerch, J. P. \& Evans, A. C. Cortical thickness analysis examined through power analysis and a population simulation. Neuroimage 24, 163-173 (2005).

65. Ad-Dab'bagh, Y. et al. Native space cortical thickness measurement and the absence of correlation to cerebral volume. Proceedings of the 11th Annual Meeting of the Organization for Human Brain Mapping, Toronto. Abstract 1736 (2005).

66. Pipitone, J. et al. Multi-atlas segmentation of the whole hippocampus and subfields using multiple automatically generated templates. Neuroimage 101, 494-512 (2014).

67. Sato, W. et al. Structural correlates of reading the mind in the eyes in autism spectrum disorder. Front. Hum. Neurosci. 11, 361 (2017).

68. Baas, D., Aleman, A. \& Kahn, R. S. Lateralization of amygdala activation: a systematic review of functional neuroimaging studies. Brain Res. Brain Res. Rev. 45, 96-103 (2004).

69. Costafreda, S. G., Brammer, M. J., David, A. S. \& Fu, C. H. Predictors of amygdala activation during the processing of emotional stimuli: a metaanalysis of 385 PET and fMRI studies. Brain Res. Rev. 58, 57-70 (2008).

70. Duerden, E. G., Arsalidou, M., Lee, M. \& Taylor, M. J. Lateralization of affective processing in the insula. Neuroimage 78, 159-175 (2013).

71. Benjamini, Y. \& Hochberg, Y. Controlling the false discovery rate: a practical and powerful approach to multiple testing. J. R. Stat. Soc. Ser. B 57, 289-300 (1995).

72. Baribeau, D. A. et al. Oxytocin receptor polymorphisms are differentially associated with social abilities across neurodevelopmental disorders. Sci. Rep. 7, 11618 (2017).

73. Marusak, H. A. et al. Amygdala responses to salient social cues vary with oxytocin receptor genotype in youth. Neuropsychologia 79(Part A), 1-9 (2015).

74. Puglia, M. H., Lillard, T. S., Morris, J. P. \& Connelly, J. J. Epigenetic modification of the oxytocin receptor gene influences the perception of anger and fear in the human brain. Proc. Natl. Acad. Sci. USA 112, 3308-3313 (2015).

75. Luo, S. et al. Interaction between oxytocin receptor polymorphism and interdependent culture values on human empathy. Soc. Cogn. Affect. Neurosci. 10, 1273-1281 (2015).

76. Tost, $\mathrm{H}$. et al. Neurogenetic effects of OXTR rs2254298 in the extended limbic system of healthy Caucasian adults. Biol. Psychiatry 70, e37-e39 (2011). author replye41-2.

77. Furman, D. J., Chen, M. C. \& Gotlib, I. H. Variant in oxytocin receptor gene is associated with amygdala volume. Psychoneuroendocrinology 36, 891-897 (2011).

78. Inoue, $\mathrm{H}$. et al. Association between the oxytocin receptor gene and amygdalar volume in healthy adults. Biol. Psychiatry 68, 1066-1072 (2010).

79. Wang, J. et al. Relationship of a common OXTR gene variant to brain structure and default mode network function in healthy humans. Neuroimage 147, 500-506 (2017)
80. Tost, H. et al. A common allele in the oxytocin receptor gene (OXTR) impacts prosocial temperament and human hypothalamic-limbic structure and function. Proc. Natl. Acad. Sci. USA 107, 13936-13941 (2010)

81. van der Meer, J. M. et al. Are autism spectrum disorder and attention-deficit/ hyperactivity disorder different manifestations of one overarching disorder? Cognitive and symptom evidence from a clinical and population-based sample. J. Am. Acad. Child Adolesc. Psychiatry 51, 1160-72 e3 (2012).

82. Demopoulos, C., Hopkins, J. \& Davis, A. A comparison of social cognitive profiles in children with autism spectrum disorders and attention-deficit/ hyperactivity disorder: a matter of quantitative but not qualitative difference? J. Autism Dev. Disord. 43, 1157-1170 (2013).

83. Lionel, A. C. et al. Rare copy number variation discovery and cross-disorder comparisons identify risk genes for ADHD. Sci. Transl. Med. 3, 95ra75 (2011).

84. Hus, V., Bishop, S., Gotham, K., Huerta, M. \& Lord, C. Factors influencing scores on the Social Responsiveness Scale. J. Child Psychol. Psychiatry 54, 216-224 (2013).

85. Charman, T. et al. Efficacy of three screening instruments in the identification of autistic-spectrum disorders. Br. J. Psychiatry 191, 554-559 (2007).

86. Chandler, S. et al. Validation of the social communication questionnaire in a population cohort of children with autism spectrum disorders. J. Am. Acad. Child Adolesc. Psychiatry 46, 1324-1332 (2007).

87. Hedrick, A. et al. Autism risk gene MET variation and cortical thickness in typically developing children and adolescents. Autism Res. 5, 434-439 (2012).

88. Tu, P. C. et al. Structural and functional correlates of a quantitative autistic trait measured using the social responsive scale in neurotypical male adolescents. Autism Res. 9, 570-578 (2016).

89. Wallace, G. L. et al. Distinct cortical correlates of autistic versus antisocial traits in a longitudinal sample of typically developing youth. J. Neurosci. 32, 4856-4860 (2012).

90. Prigge, M. B. D. et al. Social Responsiveness Scale (SRS) in relation to longitudinal cortical thickness changes in autism spectrum disorder. J. Autism Dev. Disord. 48, 3319-3329 (2018).

91. Thye, M. D., Murdaugh, D. L. \& Kana, R. K. Brain mechanisms underlying reading the mind from eyes, voice, and actions. Neuroscience $\mathbf{3 7 4}, 172-186$ (2018).

92. Yin, S., Fu, C. \& Chen, A. The structural and functional correlates underlying individual heterogeneity of reading the mind in the eyes. Biol. Psychol. 138, 179-184 (2018).

93. Schiffer, B., Pawliczek, C., Muller, B. W., Gizewski, E. R. \& Walter, H. Why don't men understand women? Altered neural networks for reading the language of male and female eyes. PLOS ONE 8, e60278 (2013).

94. Sato, W. et al. Structural neural substrates of reading the mind in the eyes. Front. Hum. Neurosci. 10, 151 (2016)

95. Sato, W., Toichi, M., Uono, S. \& Kochiyama, T. Impaired social brain network for processing dynamic facial expressions in autism spectrum disorders. BMC Neurosci. 13, 99 (2012).

96. Moor, B. G. et al. Neurodevelopmental changes of reading the mind in the eyes. Soc. Cogn. Affect. Neurosci. 7, 44-52 (2012).

97. Overgaauw, S., van Duijvenvoorde, A. C., Gunther Moor, B. \& Crone, E. A. A longitudinal analysis of neural regions involved in reading the mind in the eyes. Soc. Cogn. Affect. Neurosci. 10, 619-627 (2015).

98. Moore, W. E. 3rd et al. Facing puberty: associations between pubertal development and neural responses to affective facial displays. Soc. Cogn. Affect. Neurosci. 7, 35-43 (2012).

99. Shaw, P. et al. Intellectual ability and cortical development in children and adolescents. Nature 440, 676-679 (2006)

100. Weidt, $\mathrm{S}$. et al. Common and differential alterations of general emotion processing in obsessive-compulsive and social anxiety disorder. Psychol. Med. 46, 1427-1436 (2016).

101. Saito, Y. et al. Neural correlate of autistic-like traits and a common allele in the oxytocin receptor gene. Soc. Cogn. Affect. Neurosci. 9, 1443-1450 (2014). 